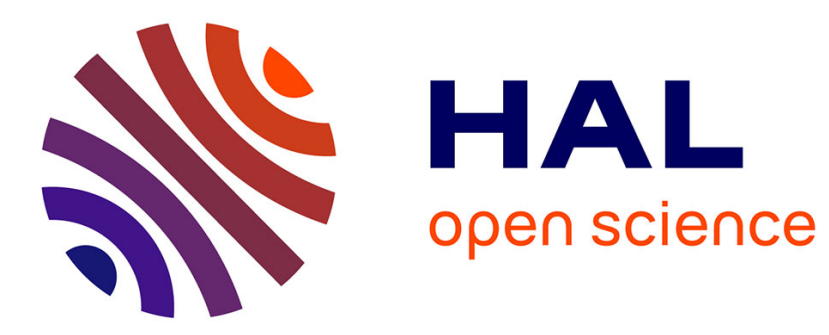

\title{
Intervenir sur les souffrances au travail : acteurs et enjeux dans la durée
}

Philippe Sarnin, Marc-Eric Bobillier-Chaumon, Bruno Cuvillier, Michèle Grosjean

\section{- To cite this version:}

Philippe Sarnin, Marc-Eric Bobillier-Chaumon, Bruno Cuvillier, Michèle Grosjean. Intervenir sur les souffrances au travail: acteurs et enjeux dans la durée. Bulletin de psychologie, 2012, 3 (65), pp.251-262. hal-00874460

\section{HAL Id: hal-00874460 https://hal.science/hal-00874460}

Submitted on 10 Jul 2017

HAL is a multi-disciplinary open access archive for the deposit and dissemination of scientific research documents, whether they are published or not. The documents may come from teaching and research institutions in France or abroad, or from public or private research centers.
L'archive ouverte pluridisciplinaire HAL, est destinée au dépôt et à la diffusion de documents scientifiques de niveau recherche, publiés ou non, émanant des établissements d'enseignement et de recherche français ou étrangers, des laboratoires publics ou privés. 


\section{INTERVENIR SUR LES SOUFFRANCES AU TRAVAIL : ACTEURS ET ENJEUX DANS LA DURÉE}

\section{Philippe Sarnin et al.}

\section{Groupe d'études de psychologie | Bulletin de psychologie}

\section{2/3 - Numéro 519}

pages 251 à 261

\section{ISSN 0007-4403}

Article disponible en ligne à l'adresse:

http://www.cairn.info/revue-bulletin-de-psychologie-2012-3-page-251.htm

Pour citer cet article :

Sarnin Philippeet al., « Intervenir sur les souffrances au travail : acteurs et enjeux dans la durée », Bulletin de psychologie, 2012/3 Numéro 519, p. 251-261.

Distribution électronique Cairn.info pour Groupe d'études de psychologie.

(c) Groupe d'études de psychologie. Tous droits réservés pour tous pays.

La reproduction ou représentation de cet article, notamment par photocopie, n'est autorisée que dans les limites des conditions générales d'utilisation du site ou, le cas échéant, des conditions générales de la licence souscrite par votre établissement. Toute autre reproduction ou représentation, en tout ou partie, sous quelque forme et de quelque manière que ce soit, est interdite sauf accord préalable et écrit de l'éditeur, en dehors des cas prévus par la législation en vigueur en France. II est précisé que son stockage dans une base de données est également interdit. 


\section{SARNIN Philippe* BOBILLIER-CHAUMON Marc-Eric* CUVILLIER Bruno* GrosJEAn Michèle**}

\section{Intervenir sur les souffrances au travail : acteurs et enjeux dans la durée}

La santé psychique au travail fait l'objet, depuis plusieurs années, de débats entre chercheurs, porteurs de théories sensiblement différentes, au sein de la psychologie du travail. D'une part, des études à l'échelle de l'organisation essaient de caractériser et d'expliquer, à partir de données quantitatives, les facteurs qui conduisent à différentes formes de souffrance chez les travailleurs (stress, anxiété, dépression, suicide). L'organisation du travail imposée aux salariés apparaît alors comme un déterminant essentiel, mais avec des variations liées aux caractéristiques individuelles. D'autre part, des études centrées sur l'activité réelle de petits groupes de travailleurs, tout en constatant, également, les incidences de l'organisation du travail, essaient de dégager les éléments qui peuvent redonner, aux collectifs de travail, du pouvoir d'agir sur cette organisation. Cependant, ces deux perspectives, psychologie des organisations et clinique de l'activité, semblent exclusives l'une de l'autre, à la fois sur le plan des méthodes (auto-questionnaires, analyses quantitatives d'un côté ; observations de l'activité réelle et analyses plus qualitatives, de l'autre côté) et, sur le plan théorique (conception du travailleur comme un être subissant et réagissant à divers facteurs ou conception du travailleur comme créateur, être en développement). Ajoutée à cela, la notion de « risque psychosocial », qui correspond plutôt à une perspective épidémiologique ou calquée sur les démarches de prévention des risques chimiques ou physiques et est venue perturber 1'appréhension des souffrances au travail, en introduisant des pratiques succinctes «d'évaluation des risques» et une modélisation de l'action préventive, sous les termes de « prévention tertiaire » (prise en charge, par des psychologues, des personnes souffrantes), «prévention secondaire» (renforcement par la formation des capacités des individus à faire face) et «prévention primaire» (action sur les facteurs de « risque »).

Nous proposons dans cet article, à partir de nos expériences de recherche-intervention en entreprise, d'essayer de clarifier les rapports entre ces différentes conceptions et de redéfinir les dispositifs de prévention des différentes formes de souffrance au travail.

À partir d'une première intervention réalisée, en 2001, avec une grande entreprise de la mécanique, qui venait d'être acquise par un groupe international du même secteur, nous ${ }^{1}$ avons pu mettre en place une collaboration régulière avec les services autonomes de santé au travail de cette entreprise. Cette collaboration, qui continue actuellement, a permis de réaliser plusieurs recherches approfondies, à base d'observations et d'enquêtes, sur différents thèmes : perception du rachat de l'entreprise et conséquences quant à la souffrance ; charge de travail et activités des ingénieurs de bureaux d'études; difficultés de communication dans les équipes de travail semiautonomes; intégration des technologies de l'information et de la communication par les salariés ; mise en place d'un observatoire collectif et d'outils de diagnostic pour le repérage des situations individuelles de souffrance psychologique.

À partir de cette série de travaux, des démarches mises en œuvre et des résultats qui en sont issus, à la fois, du côté de la recherche, mais aussi du côté des pratiques de l'entreprise, nous souhaitons analyser plus particulièrement les aspects suivants :

- la prise en compte progressive, par les acteurs de l'entreprise, des dimensions psychosociales du travail, depuis une situation de crise, d'abord, jusqu'à l'intégration dans les politiques et pratiques

\footnotetext{
* Institut de psychologie, Laboratoire GRePS, Université de Lyon.

** Institut de psychologie, Laboratoire ICAR, Université de Lyon.

Correspondance : Philippe Sarnin, Institut de psychologie, Université de Lyon, 69676 Bron cedex.

$<$ Philippe.Sarnin@univ-lyon2.fr>

1. Les auteurs de cet article, avec l'aide d'étudiants en master psychologie du travail et des organisations. Des conventions de recherche ainsi que l'accueil de stagiaires ont permis cette collaboration avec l'entreprise.
} 
quotidiennes : les moments-clés de cette évolution, les enjeux qui peuvent bloquer ce passage et ceux qui le favorisent, le rôle et la place des différents acteurs internes, le poids des facteurs extérieurs ;

- la place des interventions psychosociologiques, par rapport aux acteurs internes (CHSCT, Services de santé au travail) et la construction d'une coopération, fondée sur des échanges réciproques. Les réflexions développées ont pu également susciter, de façon plus autonome, des changements et des innovations dans les pratiques de ces acteurs, dont nous examinerons les formes ;

- les relations plus complexes rencontrées, par rapport aux gestionnaires, en particulier ceux chargés des « ressources humaines », par rapport à leur compréhension de l'activité de travail, des effets psychiques de leurs indicateurs de gestion et de leur analyse des « risques » psychosociaux.

L'objectif est de contribuer à mieux définir, dans les pratiques d'intervention, les repères qui permettent d'éviter les deux écueils de la dénonciation contemplative et de l'adhésion sans critique, par rapport aux pratiques des entreprises. La position adoptée, dès le départ, n'est pas celle de l'expert, ni celle du consultant. Bien avant que les questions sur les «risques psychosociaux» se posent dans l'espace médiatique, il s'agissait de travailler avec les acteurs de l'entreprise, pour rendre visibles les conséquences psychologiques des réorganisations en cours pour les salariés et de contribuer à redonner, au travail et au rapport des salariés à ce travail, une place dans les réflexions et les démarches engagées dans l'entreprise. Au-delà de la conscience du chercheur en psychologie du travail et des organisations, quant aux enjeux psychologiques du travail, se pose, en effet, la question du partage de ces préoccupations avec les acteurs chargés de prescrire les tâches, d'organiser le travail, afin qu'ils les prennent mieux en compte dans leurs choix organisationnels.

\section{INTERVENIR SUR LES SOUFFRANCES AU TRAVAIL}

La littérature scientifique, relative aux pratiques d'intervention sur les questions de stress, de « risques psychosociaux » (pour une synthèse, voir Rouat, 2010), tend à mettre en avant un certain nombre de conditions pour assurer l'efficacité de ce type d'interventions, lorsqu'elles portent directement sur l'organisation du travail: un engagement fort de la direction; la participation active des acteurs ; un groupe stable, pour soutenir l'intervention ; un diagnostic quantifié de la situation; la mise en œuvre d'actions planifiées ; l'évaluation et le suivi de ces actions (Saint-Arnaud, Gignac, Gourdeau, Pelletier, Vézina, 2010, p. 3). Lamontagne, Keegel, Louie, Ostry et Landsbergis (2007) montrent, dans une méta-analyse de la littérature sur l'évaluation des pratiques d'intervention, que les interventions systémiques, à l'échelle de l'organisation, sont d'autant plus efficaces qu'elles visent à transformer le travail et pas seulement à faciliter l'adaptation des individus à des conditions de travail dégradées. Pour autant, les travaux dominants, en nombre, traitent surtout des interventions centrées sur le renforcement des capacités d'adaptation des salariés, plutôt que sur des modifications à l'échelle de l'organisation. Giga Cooper et Faragher (2003) ont montré que les programmes de relaxation, les interventions, fondées sur les thérapies cognitivo-comportementales, les formations au stress ou les programmes d'aide aux employés, étaient beaucoup plus fréquents que les interventions sur l'organisation et le travail. Depuis, et face à la faible efficacité des pratiques centrées sur les individus, les interventions organisationnelles sont plus nombreuses (Lamontagne et coll., 2007), mais leurs résultats ne sont pas toujours à la hauteur des objectifs de départ (Hansez, Bertrand, Barbier, 2009). Selon Cox, Taris et Nielsen (2010, p. 217), la multiplication des démarches, pour renforcer le bien-être des travailleurs, ne permet pas de clarifier la question. Le décalage de pertinence, entre les dispositifs semi-expérimentaux des chercheurs et les réalités des organisations, est également mentionné par les mêmes auteurs. Karnas et Hellemans (2002) montrent les limites des conceptions psycho-organisationnelles et en appellent, ainsi, à des analyses du stress plus « concrètes » (p. 188).

La lecture des travaux internationaux sur les pratiques d'intervention, visant à diminuer le stress des travailleurs ou à améliorer le bien-être au travail (Aucouturier, 2010), nous montre, en effet, deux écueils :

- l'absence fréquente de prise en compte de l'activité réelle des travailleurs. Les dispositifs mis en place reposent sur des théorisations générales des travailleurs (engagement, motivation, stratégies de faire face, voire personnalité), mais sont très rarement élaborés à partir d'une analyse du travail de ceux-ci ;

- malgré le rôle reconnu du management, de l'organisation globale de l'entreprise, les actions, plus centrées sur l'encadrement (du premier niveau jusqu'au «top management»), restent, également, peu fréquentes, comme si les marges de manœuvre des managers étaient forcément limitées (par la «mondialisation», la «concurrence », etc.), alors que c'est à ce niveau qu'elles sont les plus nombreuses comme le montrent, par exemple, toutes les études utilisant le modèle de Karasek, où l'encadrement apparaît comme la catégorie ayant le plus de latitude de décision sur son activité. 
En conséquence, plusieurs questions nous apparaissent devoir être documentées. Comment construire, avec les acteurs du management, des représentations, qui intègrent l'activité des opérateurs (Lhuilier, 2009)? Comment intervenir et développer l'action sur le travail à l'échelle de l'organisation et pas seulement dans tel ou tel atelier ou service ? Comment aider les acteurs à raisonner en termes de "ressources ", plutôt que de « risques » (Clot, 2010, p. 102) ?

Nous souhaitons ainsi, à partir d'un retour réflexif sur les démarches construites progressivement avec les acteurs, apporter quelques éléments de réponse fondés sur les découvertes, mais, aussi, les erreurs d'un cheminement guidé par la recherche d'une meilleure compréhension des conditions par lesquelles une organisation peut «mûrir» (voir Rouat, 2010) vers la prise en compte, à tous les niveaux, des possibles souffrances liées au travail.

\section{ANNÉES D'INTERVENTIONS}

Cette entreprise française, qui comprend 14000 salariés dont 11000 en France, a été achetée en 2001 par une entreprise scandinave du même secteur d'activités. Dans les mois qui ont suivi ce rachat, les médecins du travail ont observé, lors des visites médicales obligatoires, que de plus en plus de salariés se trouvaient en difficulté dans leur travail : une pression importante, une charge de travail croissante, des interrogations sur l'avenir les préoccupaient à un point tel que nombre d'entre eux «craquaient», s'effondraient en pleurs dans le cabinet médical. En accord avec le comité d'hygiène, de sécurité et des conditions de travail (CHSCT), l'équipe médicale a proposé qu'une étude soit conduite afin d'évaluer plus précisément l'ampleur du phénomène au niveau de l'établissement regroupant les fonctions tertiaires de l'entreprise (commercial, bureaux d'études, finances, etc.). La première demande, exprimée par les médecins et discutée avec le CHSCT, qui a été adressée à notre équipe universitaire correspondait à la volonté d'établir un état des lieux, à l'aide « d'instruments validés scientifiquement, du stress, de l'anxiété et de la dépression ». Nous avons rapidement transformé cette recherche d'expertise en un questionnement en commun avec les médecins du travail, le CHSCT et les responsables de l'établissement sur les préoccupations de l'entreprise, l'enjeu du rachat et les inquiétudes et réactions des salariés.

Nous avons construit avec les services de santé au travail un dispositif de mesure, d'abord à partir d'une enquête ponctuelle en 2001, puis sous la forme d'un «observatoire » permanent implanté en 2006 dans l'établissement tertiaire et généralisé, en 2008, à l'ensemble des établissements de l'entreprise situés en France. Ce dispositif d'observatoire permet, lors des visites médicales et à partir du remplissage d'un questionnaire sur un poste informatique, de recueillir en permanence des données sur la perception des salariés pour différents indicateurs : stress, dépression, anxiété, reconnaissance, conditions de travail, etc. tout en offrant un outil de diagnostic individuel utilisé par les médecins lors de la visite obligatoire. Pendant la même période, une série de recherches plus précises a permis d'approcher la réalité du travail à partir de questionnements issus soit de problématiques repérées par les médecins soit de demandes exprimées par des responsables de services ou d'ateliers ou par les représentants des salariés dans le cadre de leurs activités mutualistes. La prise en charge individuelle par les médecins les amène également à faire évoluer les instruments utilisés pour les évaluations collectives, tandis que les outils de mesure de l'observatoire leur permettent de repérer des travailleurs en difficultés. Les deux alimentent les réflexions des acteurs sur le travail et son organisation dans les équipes de travail.

Le tableau 1 synthétise les différentes opérations conduites par l'équipe de chercheurs avec l'aide d'étudiants, certaines à l'échelle de l'ensemble de l'organisation, d'autres auprès de collectifs de travail particuliers. Bien sûr, dans cette grande entreprise, d'autres actions ont été menées sans notre concours : un programme de formation ambitieux pour l'encadrement et les représentants du personnel, des conférences, des pages dédiées aux conditions de travail et au stress sur le site intranet de l'entreprise, diverses interventions d'ergonomes, de psychologues, etc. Un service "santé et bien-être », rattaché à la direction des ressources humaines, a, en effet, été créé dans le cadre d'une politique générale de la société scandinave qui a racheté l'entreprise.

Le groupe, qui constitue le noyau central de ces travaux, comprend la dizaine de médecins du travail des différents établissements, la responsable du service "santé et bien-être », qui se réunissent très habituellement, et les chercheurs. Les travaux de ce groupe font régulièrement l'objet de présentations et sont débattus avec les représentants du personnel (comités d'entreprise et CHSCT des différents établissements) et l'encadrement. Les actions plus ponctuelles mobilisent les acteurs concernés.

Les méthodes mise en œuvre combinent l'utilisation de différents instruments de recherche, en fonction du niveau de l'étude : questionnaires et échelles pour les analyses au niveau de l'organisation, entretiens, observations directes et « armées » (logiciels de suivi d'activité, grilles d'observations, enregistrements audiovisuels) de fonctionnements collectifs ou d'activités. Les résultats obtenus sur chaque chantier de recherche alimentent les autres études dans leurs questionnements, mais, aussi, pour faire évoluer les instruments utilisés. Il s'agit de jouer, à la fois, sur la triangulation des 
problématiques (local/global) et la triangulation des méthodes (par exemple, intégration dans le questionnaire général de nouvelles questions issues des chantiers locaux) pour accroître la validité et la richesse des analyses et des propositions d'action.

\section{LES DIFFÉRENTS « CHANTIERS »}

Nous évoquerons, dans cette partie, quelques éléments saillants, mis en évidence par les différents travaux conduits dans l'entreprise. Le tableau 1 résume les principales caractéristiques des études sur lesquelles nous appuyons notre réflexion.

\begin{tabular}{|c|c|c|c|c|c|}
\hline Étude & Période & $\begin{array}{l}\text { Acteurs } \\
\text { enquêtés }\end{array}$ & $\begin{array}{l}\text { Entretiens } \\
\text { individuels }\end{array}$ & Questionnaires & $\begin{array}{l}\text { Observations } \\
\text { directes }\end{array}$ \\
\hline $\begin{array}{l}\text { Première enquête suite } \\
\text { au rachat de l'entreprise, } \\
\text { à la demande des } \\
\text { médecins du travail }\end{array}$ & $2000-2001$ & Services tertiaires & $N=21$ & $\begin{array}{l}\mathrm{N}=416 \\
\text { Questions ad hoc } \\
\text { et échelle MSP }\end{array}$ & \\
\hline $\begin{array}{l}\text { Étude à la demande du } \\
\text { service « achats » }\end{array}$ & $2001-2002$ & $\begin{array}{l}\text { Service achats } \\
\text { "global sourcing» }\end{array}$ & $N=6$ & $N=38$ & $\begin{array}{l}\text { - Six salariés } \\
\text { observés pendant } \\
\text { deux jours chacun } \\
\text { - Deux réunions de } \\
\text { travail vidéoscopées }\end{array}$ \\
\hline $\begin{array}{l}\text { Étude sur la charge de } \\
\text { travail des ingénieurs } \\
\text { d'études }\end{array}$ & $2003-2004$ & $\begin{array}{l}\text { Ingénieurs d'un } \\
\text { bureau d'études }\end{array}$ & $N=12$ & & $\begin{array}{l}\text { - Dix ingénieurs } \\
\text { observés pendant une } \\
\text { journée chacun } \\
\text { - Observations des } \\
\text { réunions de service }\end{array}$ \\
\hline $\begin{array}{l}\text { Première phase de test } \\
\text { de l'observatoire du } \\
\text { stress }\end{array}$ & $\begin{array}{l}\text { À partir de } \\
2006 \text { jusqu'à } \\
\text { début } 2008\end{array}$ & Services tertiaires & $\begin{array}{l}\text { (entretiens pour } \\
\text { mettre au point le } \\
\text { dispositif) }\end{array}$ & $N=278$ & \\
\hline $\begin{array}{l}\text { Échelles MSP, Cohen, } \\
\text { HAD, Karasek et } \\
\text { questions spécifiques }\end{array}$ & $\begin{array}{l}\text { Étude sur } \\
\text { l'usage des } \\
\text { mails par les } \\
\text { salariés }\end{array}$ & 2006-2007 & $\begin{array}{l}\text { Bureau d'études } \\
\text { «électricité- } \\
\text { électronique » }\end{array}$ & $N=18$ & $\begin{array}{l}\text { - Auto-observations } \\
\text { des mails par } 18 \text { salariés } \\
\text { - Cinq observations } \\
\text { avec verbalisations } \\
\text { simultanées lors du } \\
\text { traitement des mails }\end{array}$ \\
\hline $\begin{array}{l}\text { Étude de la } \\
\text { communication dans les } \\
\text { équipes } \\
\text { semi-autonomes de } \\
\text { production }\end{array}$ & $2007-2008$ & $\begin{array}{l}\text { Équipes } \\
\text { semi-autonomes } \\
\text { de travail en } \\
\text { fabrication }\end{array}$ & & $N=46$ & $\begin{array}{l}\text { - Observation des } \\
\text { relèves } \\
\text { - Analyse des traces }\end{array}$ \\
\hline $\begin{array}{l}\text { Deuxième phase de } \\
\text { l'observatoire du stress : } \\
\text { généralisation à toute } \\
\text { l'entreprise }\end{array}$ & $\begin{array}{l}\text { À partir } \\
\text { de } 2008\end{array}$ & $\begin{array}{l}\text { Ensemble des } \\
\text { salariés de } \\
\text { l'entreprise }\end{array}$ & $\begin{array}{l}\text { (entretiens pour } \\
\text { mettre au point le } \\
\text { dispositif) }\end{array}$ & $\begin{array}{l}\mathrm{N}=7500 \text { fin } 2010 \\
\text { Échelles : Cohen, } \\
\text { HAD, Karasek, } \\
\text { Siegrist et questions } \\
\text { spécifiques }\end{array}$ & \\
\hline
\end{tabular}

\begin{tabular}{|c|c|c|c|c|c|}
\hline $\begin{array}{l}\text { Étude sur les problèmes } \\
\text { posés par l'usage de } \\
\text { l'anglais dans } \\
\text { l'entreprise }\end{array}$ & $2009-2010$ & $\begin{array}{l}\text { Bureau d'études } \\
\text { «moteurs» }\end{array}$ & $N=18$ & & $\begin{array}{l}\text { Cinq salariés observés } \\
\text { pendant une journée } \\
\text { chacun }\end{array}$ \\
\hline $\begin{array}{l}\text { Étude sur les } \\
\text { conséquences du } \\
\text { chômage technique de } \\
\text { longue durée (à la } \\
\text { demande de la mutuelle } \\
\text { des salariés) }\end{array}$ & $2010-2011$ & $\begin{array}{l}\text { Ouvriers et } \\
\text { employés de } \\
\text { profuction }\end{array}$ & $N=23$ & $\begin{array}{l}\mathrm{N}=102 \\
\text { Questionnaire } \\
\text { spécifique sur la } \\
\text { perception du } \\
\text { chômage partiel et } \\
\text { les conséquences } \\
\text { sur la vie } \\
\text { professionnelle et } \\
\text { la vie personnelle }\end{array}$ & \\
\hline
\end{tabular}

Tableau 1. Principales caractéristiques des études effectuées. 
Le rachat de l'entreprise et la construction sociale du stress

La première étude conduite reposait sur une série d'entretiens semi-directifs, qui ont permis la construction d'un questionnaire, diffusé auprès des salariés de l'établissement tertiaire. Combinant des échelles de mesure du stress (MSP $25^{2}$ de Lemyre, Tessier, Fillion, 1990) et des questions sur les préoccupations des salariés (rachat de l'entreprise, passage aux 35 heures), la charge de travail, les relations avec les collègues et la hiérarchie, les conditions de travail à améliorer.

L'analyse a montré un phénomène remarquable, lié au rachat de l'entreprise. L'échelle de stress indiquait un niveau général élevé, qui semblait confirmer les hypothèses des médecins. Les interprétations, permises par les analyses statistiques (Sarnin, 2003; Sarnin, Fernandez, Malaurie, 2006) et les entretiens avec les salariés, nous ont orientés vers l'idée d'une construction sociale d'une perception excessive, peu connectée à la réalité de ce rachat. En fait, devant l'absence de discours sur le devenir de l'organisation, de la part de l'entreprise scandinave, et le sentiment d'avoir été abusé par des discours antérieurs de la direction, du type : " l'entreprise n'est pas à vendre », l'interprétation de la situation, répandue parmi les salariés, a été celle d'une compétition entre les salariés français et ceux de l'entreprise scandinave qui fabriquent les mêmes produits. « Il faut qu'on soit meilleurs qu'eux sinon on va disparaître » résume, assez bien, cette représentation. L'année qui a suivi le rachat, les résultats économiques de l'entreprise française ont été largement meilleurs, par rapport aux objectifs initiaux, mais aussi par rapport aux résultats de l'année de l'entreprise scandinave. Cette dernière, dont la motivation des dirigeants n'était pas la disparition de l'entreprise française, mais une politique d'expansion externe et de présence sur les marchés mondiaux, n'a donc pas eu à regretter cet achat. La souffrance, ressentie par les salariés, était ainsi, en partie, générée par l'interprétation d'une situation de forte incertitude (Lancry, 2007), ayant généré des exigences excessives parmi les salariés.

\section{Le travail d'articulation des acheteurs}

Les tensions ressenties par les salariés, en suite du rachat de l'entreprise, se sont particulièrement manifestées chez les acheteurs. Le service comprend une dizaine de personnes, mais se situe au cœur des interactions entre les différentes unités

2. MSP 25 : Mesure du stress psychologique, échelle de 25 items. de production, les bureaux d'études, les fournisseurs et l'entreprise scandinave (recherche d'économies d'échelle). L'objectif d'une observation du travail collectif et de l'analyse de l'activité des acheteurs (Dupont, 2002) était de comprendre ce travail d'articulation (Strauss, 1992) dans le cadre de projets complexes et très instables. Par touches progressives, ce travail d'articulation ne semblait plus pouvoir être piloté et maîtrisé par les salariés, dont l'activité était structurée, essentiellement par des attracteurs cognitifs (objets, écrits, évènements qui influencent le cours d'action, voir Lahlou, 2000).

\section{La fragmentation du travail des ingénieurs de bureau d'études}

En observant 10 ingénieurs d'un bureau d'études, pendant des journées complètes (Sarnin, Balas-Broche, 2006), ce chantier a permis de mettre en évidence la fragmentation des activités de ces salariés, censés pouvoir dégager des temps de réflexion suffisants pour travailler sur leurs projets. Les interruptions et la place grandissante des outils de collaboration à distance (visioconférences, audioconférences, etc.) entraînent une charge de travail très lourde. Bien qu'ils soient des ingénieurs centrés sur des questions très techniques, l'essentiel de leurs activités s'apparente à du management d'informations, de personnes, de fournisseurs, de la «débrouille», avec des horizons temporels et des objectifs, qui se superposent. Malgré une autonomie affirmée dans la conduite des projets, l'observation montre de nombreuses dépendances et contraintes, qui leur laissent le sentiment de ne pas faire du «bon boulot ».

\section{Les débordements du courrier électronique}

Bien que l'usage du courrier électronique soit devenu, depuis quelques années, un élément essentiel du travail, les plaintes à son sujet ont conduit à examiner de plus près cet usage (Sornet, 2007). Les observations réalisées ont relevé les stratégies utilisées par les salariés pour gérer les flux de courriels, qui les conduisent à re-planifier régulièrement leur activité, mais, aussi, le fait qu'ils soient devenus un vecteur de communication dans les collectifs de travail et plus seulement un système de transmission d'information. L'ampleur des débordements sur le hors-travail a conduit, ensuite, l'entreprise à élaborer, à partir de cette étude, quelques règles générales «opposables » (vis-à-vis d'un supérieur ou d'un autre service), qui sont, avec le recul, effectivement mobilisées par les salariés : limitation des horaires d'envoi, meilleur ciblage dans la diffusion, éviter les demandes de réponse immédiate, etc. 


\section{Les équipes semi-autonomes et la surabon- dance d'informations}

L'histoire de cette entreprise est marquée, à la fois, par une présence syndicale forte et par de nombreuses expérimentations en matières d'organisation et de conditions de travail. Ainsi, les unités de production sont, depuis longtemps, organisées, à partir du modèle de l'équipe semi-autonome, développé par le courant sociotechnique avec, cependant, des différences importantes: si les équipes sont relativement autonomes dans l'activité, dans chaque équipe un chef existe, qui est présenté comme un «manager». Les équipes disposent de salles de réunions et ont de nombreuses informations sur leurs activités (production, qualité, conditions de travail, accidents, etc.). La question posée par les acteurs de l'entreprise portait sur la communication entre chefs et équipes (Digonnet, 2008). En effet, malgré une volonté de la direction de favoriser les échanges dans les collectifs de travail, de façon à ce que les problèmes rencontrés dans l'activité soient traités par l'équipe elle-même, il semblait que les éléments d'information, fournis avec abondance à ces équipes et affichés en salle de réunion, ne servaient pas à faciliter ces échanges. Les observations des réunions, l'analyse des informations transmises et discutées indiquaient, avec des variations selon les équipes, des échanges créatifs mais peu d'utilisation de l'information fournie, ainsi qu'une charge de travail importante pour les chefs, liée à la fabrication de ces informations, au détriment des échanges directs avec les ouvriers.

\section{L'usage de l'anglais et la reconnaissance professionnelle}

En raison du rachat par une entreprise scandinave, l'usage de l'anglais a été généralisé dans les échanges internes, particulièrement pour l'encadrement. Ce sont, aussi, les outils de travail, comme les logiciels techniques, les rapports, les dossiers techniques, notes de service, informations générales, qui font de moins en moins l'objet de traductions. Un programme important de formation à l'anglais est proposé aux salariés pour accompagner cette évolution : les personnes nouvellement recrutées doivent posséder un niveau minimum dans cette langue. Cependant, cet usage, de plus en plus massif, est apparu aussi comme une source de souffrance pour certains salariés. L'étude effectuée (Krief, 2010) combinait l'observation de l'usage de l'anglais au cours de journées de travail habituelles et des entretiens approfondis avec des salariés de différents niveaux et catégories. Si pour les personnels les plus diplômés ou jeunes, l'usage de l'anglais est peu problématique, les moins qualifiés, les ouvriers en particulier, n'ont pas à l'utiliser. Le problème se pose surtout pour les salariés anciens, ouvriers qualifiés ou techniciens, dont les compétences sont reconnues, mais qui se trouvent en difficulté lorsqu'ils doivent lire, écouter ou parler anglais. Les stratégies développées visent à augmenter les ressources (fabrication collective de lexiques techniques, recherche de collègues maîtrisant l'anglais, utilisation de sites internet de traduction, etc.) ou à fuir les situations où l'anglais est indispensable (réunions, audioconférences, etc.). L'accroissement de la charge de travail lié à cet usage n'est pas négligeable : efforts cognitifs, temps de traduction, erreurs et rattrapages à la suite d'incompréhensions ${ }^{\mathrm{TM}}$ Pour les plus anciens des personnels qualifiés, qui ne maîtrisent pas du tout cette langue, l'enjeu est la disparition de la reconnaissance de leurs qualités professionnelles : ne pas pouvoir se faire comprendre, exprimer ses idées, ne plus pouvoir discuter du métier avec certains collègues sont des éléments très déstabilisateurs. Les plus âgés adoptent des stratégies d'évitement «en attendant la retraite», ceux qui ont encore 10 ou 15 ans d'activité devant eux expriment une forte souffrance.

\section{Les conséquences du chômage technique de longue durée}

Pendant l'été 2008, le carnet de commandes de l'entreprise s'est effondré de $60 \%$. Fin 2008, les personnes en contrat à durée déterminée et les intérimaires n'ont pas été réembauchés. À partir de janvier 2009, et avec l'aide de l'État, deux jours de chômage technique par semaine ont été mis en place dans la plupart des secteurs jusqu'à l'été 2010. La crise économique et ce chômage technique de très longue durée ont préoccupé les dirigeants de la mutuelle d'entreprise des ouvriers et employés. Sans obtenir le soutien de la direction des ressources humaines, mais en relation avec le service de santé au travail, cette mutuelle nous a sollicités pour une étude spécifique sur les conséquences sociales et psychologiques de ce chômage partiel (Bebessiki, 2010). L'observatoire du stress (voir ci-dessous) a montré une légère baisse des indicateurs de stress au cours de l'année 2009, tandis que ceux relatifs à l'anxiété se dégradaient en 2010, peu avant que l'activité ne reprenne totalement. En plus de l'inquiétude, liée à la situation économique, la reprise du travail signifiait, en fait, pour les ouvriers, des conditions de travail différentes de celles qu'ils avaient connues avant la crise, lorsque l'activité de l'entreprise était particulièrement importante. Travailler sans intérimaires ou personnels en contrat à durée déterminée modifie les tâches affectées et entraîne une sorte de régression des qualifications pour intégrer des tâches plus ingrates. La période de chômage partiel a créé quelques tensions entre ceux qui étaient en chômage deux jours par semaine et 
certains secteurs (pièces détachées), dont l'activité était, au contraire, très importante. Cependant, il semble que le temps disponible et les échanges sur la situation ont, plutôt, renforcé les relations entre les salariés et avec leur hiérarchie. Les deux journées libres ont, principalement, été utilisées pour des activités familiales (enfants, bricolage, etc.), qui ont favorisé le bien-être des salariés. Selon les métiers exercés, la charge de travail pouvait être importante pendant les trois jours travaillés : pour les cadres, les techniciens de bureau d'études, par exemple, la crise n'a pas des conséquences aussi directes et rapides qu'en production. Les salariés ont pu apprécier le dispositif de chômage partiel mis en place en France, alors que leurs collègues scandinaves ont subi des licenciements collectifs.

\section{«Un questionnaire peut être plus qu'un ques- tionnaire » (Kristensen, 2010)}

«L'observatoire du stress» a été construit progressivement avec les acteurs de l'entreprise et en tirant parti des autres études réalisées. L'objectif de mesure à l'échelle de l'organisation de certains indicateurs de stress, anxiété et dépression, de leurs évolutions et de leurs variations entre services, n'était pas une fin en soi. Les échanges, générés par la construction de ce dispositif, avec les représentants du personnel, l'encadrement et les services de santé au travail, ont permis de s'assurer de la pertinence des échelles utilisées et des variables prises en compte. Le travail important de restitution régulière des résultats, tous les six mois au départ, puis tous les ans à partir de 2010, s'effectue grâce à l'implication des médecins du travail, qui participent à l'animation de ces restitutions dans les différents secteurs dont ils ont la responsabilité. Une première version du dispositif a été testée dans l'établissement tertiaire, en 2006-2007. Le retour d'expérience et les débats internes, qui ont suivi, ont confirmé l'intérêt de ce dispositif, en même temps que des ajustements étaient proposés. La direction de l'entreprise a soutenu l'extension à tous les établissements de l'entreprise de cet observatoire, à partir du printemps 2008, sans limitation dans la durée. Fin décembre 2010, 7500 salariés, sur une population de 10100 , avaient répondu au questionnaire proposé.

Concrètement, cet observatoire repose sur la mise en place d'une dizaine de postes informatiques dans les services de santé au travail des différents établissements de l'entreprise. Les salariés sont invités, lors de la visite médicale obligatoire, à remplir un questionnaire, installé sur ces postes, qui comprend les éléments suivants :

- échelle de Karasek : Job Content Questionnaire (Karasek, Theorell, 1990), évaluant les dimensions
« demande psychologique », « latitude de décision » et « soutien social ». Pour faciliter les comparaisons avec des données nationales, la version de l'échelle utilisée est celle de l'enquête SUMER (Surveillance médicale des expositions aux risques) (Niedhammer, Chastang, Gendrey, David, Degioanni, 2006; Coutrot, Mermilliod, 2010) ;

- échelle HAD (Hospital Anxiety and Depression) relative aux dimensions «dépression » et « anxiété » (Mykledu, Stordall, Dahl, 2001);

- échelle de «stress perçu» de Cohen: Perceived Stress Scale (Cohen, Kamarck, Mermelstein, 1983);

- durant la phase de test, en 2006-2007 et jusqu'à l'été 2010, l'échelle de Lemyre « Mesure du stress psychologique » (MSP25, Lemyre et coll., 1990) avait été utilisée, pour permettre des comparaisons avec les résultats de l'enquête de 2001. La forte corrélation des résultats de cette échelle avec ceux de l'échelle de Cohen ( 0,75 sur un échantillon de 6643 salariés de l'entreprise) nous a conduits à la retirer du dispositif au profit de l'échelle Effort/ reward imbalance (ERI) de Siegrist (1996), qui introduit des dimensions différentes ;

- échelle de Siegrist ERI, qui évalue «l'effort extrinsèque », «l'effort intrinsèque » et les « récompenses » en retour, perçues par les salariés ;

- une série de questions, ne correspondant pas à des échelles, pour examiner différents facteurs spécifiques, susceptibles de poser problème aux salariés : horaires de travail, charge et conditions de travail, changements dans l'organisation, usage de l'anglais, conflits avec l'entourage professionnel, clarté des rôles, etc.;

- les variables sociodémographiques habituelles, telles que : âge, sexe, ancienneté, niveau de formation, statut, service, etc.

Les salariés ne sont pas obligés de répondre au questionnaire, mais il se constate que les conditions de passation entraînent très peu de refus (moins de $1 \%$ ) : l'anonymat est préservé, la passation se fait au sein du service médical, dans le respect des règles déontologiques des médecins. Un bilan individuel est imprimé après chaque passation et le logiciel se referme automatiquement, empêchant quiconque de regarder les réponses ou de réimprimer le bilan. Ce bilan est la propriété du salarié, il peut ou non en discuter, lors de la visite médicale qui suit. L'application permet, également, de remplir le questionnaire à une autre occasion que la visite médicale, si le salarié souhaite examiner l'évolution de ses résultats pour les différents indicateurs utilisés. De fait, les conditions de passation et la confiance accordée aux médecins font que plus de $95 \%$ des salariés n'hésitent pas à discuter leur bilan avec le médecin. 
L'observatoire permet, donc, deux types d'utilisation :

- à partir des bilans individuels peut s'amorcer un dialogue avec le médecin sur les conditions de travail des salariés, ainsi que le repérage et la prise en charge des personnes en grande difficulté. Les médecins ont intégré l'usage du dispositif dans leur pratique, au même titre que d'autres examens plus classiques (mesure de la tension, vue, audition, etc.). Ils ont, également, construit, à l'aide de cet observatoire, un dispositif de suivi des salariés les plus en difficultés (environ 400 en moyenne pour une population de 10100 );

- les données brutes sont conservées, en respectant l'anonymat, sur le poste informatique. Elles sont envoyées, chaque mois, à un des chercheurs de l'équipe, qui effectue des analyses statistiques, dont les résultats et interprétations sont restituées et discutées régulièrement avec les médecins, mais, aussi, dans le cadre des réunions de CHSCT. Les analyses portent sur l'évolution des indicateurs dans le temps et les variations entre les différents groupes de l'entreprise (avec un seuil minimum de 10 salariés par catégorie pour préserver l'anonymat). Dans le cadre d'une convention avec l'entreprise, ces données peuvent être exploitées pour des publications scientifiques.

Ce qui pourrait n'apparaître que comme un questionnaire de plus est, en fait, un instrument d'échanges particulièrement utile dans l'entreprise pour déclencher des actions plus concrètes, repérer des groupes en difficulté, définir des priorités et vérifier que les actions entreprises ont amélioré la situation des salariés. La construction participative $\mathrm{du}$ dispositif et les nombreuses restitutions ont, petit à petit, fait évoluer les représentations des acteurs sur les questions de stress et de souffrance au travail.

\section{ÉVOLUTIONS DES ACTEURS DE L'ENTREPRISE ET POSITIONNEMENT DES CHERCHEURS}

Les acteurs de terrain : médecins, représentants du personnel, direction générale et direction des ressources humaines, cadres opérationnels

Les médecins du travail sont les acteurs majeurs dans la réalisation de ces chantiers. Initiateurs de la démarche, la soutenant de manière constante, essayant de débloquer les premières réticences de la direction, il semble qu'ils aient trouvé, dans ces travaux, des voies de développement de leur métier (dans les termes de Clot, 2008), mais, aussi, un accroissement de légitimité au sein de l'organisation. La confiance manifestée par les salariés et les sollicitations régulières, dont ils font l'objet de la part de l'encadrement, les ont confortés en cela. Du coup, nous avons pu observer une implication croissante de ces médecins dans des activités de réflexion sur les conditions de travail et sur l'exercice de leur métier (participation à des congrès de médecine du travail, conférences dans divers organismes, formation à la clinique médicale du travail, implication dans les études épidémiologiques nationales, etc.), ce qui a, d'ailleurs, accru leur charge de travail.

Les représentants du personnel, tout en participant activement à la démarche dans le cadre de nombreuses réunions ordinaires ( $\mathrm{CE}, \mathrm{CHSCT})$ ou spécifiques (restitutions), nous sont apparus plus distants. «Avec $100 €$ de plus par mois, il n'y aura plus de stress » est une phrase entendue au début de la démarche. Après quelques années, les positions ont évolué. La démarche et, en particulier, l'observatoire du stress, fait partie maintenant du quotidien. Certains représentants syndicaux, qui avaient exprimé une hostilité au départ en s'interrogeant sur leur participation, sont maintenant actifs et impliqués, avec, bien sûr, le souci permanent de la transformation des analyses en actions concrètes. L'épisode de la demande, par la mutuelle, d'une étude sur les conséquences du chômage partiel, montre une situation un peu paradoxale. Cette mutuelle d'entreprise, qui est dirigée, en fait, par les représentants des différents syndicats de salariés de l'organisation, a transmis, ainsi, des préoccupations qui ne pouvaient être exprimées ouvertement par les syndicats à la direction, mais qui étaient réelles. Les enjeux de négociation sociale et les consignes des fédérations ne permettaient pas d'aborder ouvertement les questions de souffrance psychologique, de mesure du stress, avec la direction.

Du côté des gestionnaires des ressources humaines, les questions de stress au travail ont été abordées majoritairement avec les outils qui fondent leurs activités : formation continue (stages «gestion du stress », « affirmation de soi », etc.), évaluation (à « $\left.360^{\circ} »\right)$ et accompagnement de l'encadrement. Le délai entre la première enquête de 2001 et l'expérimentation de l'observatoire, en 2006, dans l'établissement tertiaire, s'explique, en grande partie, par les résistances des responsables des ressources humaines de l'époque, autour des difficultés à penser la discussion sur l'organisation du travail, face à la place grandissante prise par les services de santé au travail sur ces questions. Le déblocage et la généralisation de l'observatoire, en 2007-2008, sont liés à des changements de responsables à la direction des ressources humaines et à un agenda médiatique favorable à la prise de conscience des difficultés rencontrées par les salariés. Cependant, il reste beaucoup à faire pour que les dispositifs de gestion du personnel, qui visent à favoriser la mobilité ou l'adaptation des salariés 
aux évolutions du travail et de l'organisation ${ }^{3}$, soient infléchis pour mieux tenir compte du développement professionnel des individus et renforcer les collectifs de travail.

Du côté de l'encadrement, l'évolution des représentations est passée par plusieurs phases. D'abord réticents à voir les problématiques de souffrance abordées ouvertement par l'entreprise (remise en cause possible de l'organisation du travail, qu'ils ont contribué à implanter), les chantiers, conduits dans certains secteurs, les ont rassurés sur l'intérêt concret de se pencher sur ces questions, pour résoudre un certain nombre de difficultés rencontrées par les travailleurs et qui les mettent, aussi, dans l'embarras. Au début, certains ont, parfois, perçu les restitutions des données de l'observatoire comme une nouvelle forme d'évaluation de leurs compétences dans le management de leurs équipes. Cependant, les débats instaurés par la suite ont, semble-t-il, montré qu'il était possible, sans risque, d'intégrer une réflexion sur les conséquences psychologiques, pour les salariés, des choix d'organisation en discussion. Pour avoir participé sur plusieurs années à de nombreuses réunions avec l'encadrement, c'est cette évolution dans les représentations de celui-ci qui est apparue le plus nettement pour les chercheurs, par rapport aux autres catégories d'acteurs de l'entreprise mentionnées plus haut. Cependant, le développement de la «production allégée » ${ }^{4}$ dans l'entreprise remet constamment cette évolution en question, du fait des conséquences importantes de ce mode de production sur les conditions de travail (Valeyre, 2006) et des ambiguïtés autour de la prise en compte du travail réel (Bourgeois, Gonon, 2010).

\section{Le positionnement des chercheurs}

D'abord sollicités, en tant qu'experts de la psychologie du travail et des organisations, par les médecins du travail, confrontés à une souffrance croissante des salariés, la collaboration mise en place progressivement nous a permis, de façon plus «égalitaire », aussi bien de mettre à l'épreuve certains modèles théoriques préexistants, que de nous mobiliser, à partir de nouvelles questions, issus du terrain. Le partage des connaissances, parfois revendiqué par les spécialistes de la recherche-action (Liu, 1997), présente, bien sûr, des limites. La complexité d'une grande entreprise ne permet pas d'appréhender tout ce qui s'y déroule, de même que la participation ponctuelle

3. L'observatoire montre que $27,5 \%$ des salariés ont changé de poste dans l'année qui précède la passation du questionnaire et $41,4 \%$ ont changé de supérieur hiérarchique dans l'année qui précède $(\mathrm{n}=6643)$.

4. Qui essaie d'éliminer tous les déplacements, activités et gestes non productifs des travailleurs. aux débats sur le métier des acteurs ne facilite pas la transmission de connaissances académiques aux acteurs les plus à même de faire évoluer l'organisation. Cependant, notre apport de chercheurs tient, nous semble-t-il, dans cette évolution des représentations que nous avons indiquée précédemment chez ces acteurs. Dugué, Petit et Daniellou (2010) décrivent «l'intervention ergonomique comme acte pédagogique » à travers la confrontation, lors de l'intervention, de différentes rationalités. Cette évolution des représentations correspondrait au registre de l'éducation (p. 17), à travers le regard, porté par les chercheurs, sur l'existence de marges de manœuvre et les questions de santé inhérentes aux choix organisationnels. D'un autre côté, nos réflexions, au cours de cette démarche, ont renforcé nos convictions sur la complémentarité des méthodes dites quantitatives et qualitatives, pour mieux appréhender finement la réalité du travail, sans renoncer, pour autant, à des actions plus générales, à l'échelle de l'organisation. Le positionnement adopté vise, ainsi, à maintenir l'articulation entre les analyses organisationnelles et les analyses de l'activité, pour faire face aux limites des deux perspectives : risque d'abstraction stérile, d'un côté et risque d'enkystement d'expériences locales, de l'autre.

\section{DISCUSSION}

Les chantiers, dont nous avons rendu compte rapidement, ont fait l'objet ou vont faire l'objet d'autres publications. Nous avons insisté sur l'articulation entre les différents niveaux de l'organisation, de l'individu à l'ensemble de l'entreprise, en passant par les collectifs de travail. Les enjeux de telles interventions, avec toutes leurs limites, nous semblent concerner plusieurs aspects.

Sur le plan épistémologique, il s'agit d'éviter certaines dérives de la recherche en psychologie du travail et des organisations. Le souci de la mise à distance, dans de nombreuses pratiques de recherche, s'appuyant uniquement sur des questionnaires et des analyses statistiques complexes, peut aboutir à fabriquer des « construits psychologiques» plus proches des artefacts que de la compréhension des comportements concrets des travailleurs et du travail. Les évaluations contradictoires des effets d'interventions utilisant ce cadre nous semblent correspondre à cet éloignement de la réalité des représentations et comportements. Cependant, les perspectives plus cliniques de l'activité et du travail (Clot, Lhuilier, 2010) montrent, aussi, des limites. Favorisant le développement du métier, pour les travailleurs qui ont la chance de participer à ces recherches, la question de la transformation d'ensemble de l'organisation reste posée. Des rapprochements entre ces perspectives sont 
possibles, comme le montre Vézina (1999), mais l'articulation entre l'action (locale, concrète) sur le travail et la structure (globale, institutionnelle) de l'organisation doit être pensée plus fortement.

$\mathrm{Du}$ point de vue disciplinaire, nous avons, parfois, l'impression d'un découpage entre les psychologues qui s'occupent du travail, proches des travailleurs, et les psychologues centrés sur l'organisation, plus proches des directions. Nous préférerions aller au fond de l'appellation la plus générale et internationale de la discipline: « psychologie du travail et des organisations », en évitant, autant que possible, cette distinction. Il nous faut travailler avec les directions pour pouvoir infléchir leur choix organisationnels en fonction des résultats des analyses de l'activité réelle des travailleurs.

Les possibilités de développement du métier pour augmenter le pouvoir d'agir et atténuer les souffrances nous semblent, ainsi, en lien avec le développement de l'organisation. La psychologie des organisations, enrichie par la clinique de l'activité, pourrait construire des théories plus « fondées » ou « ancrées » (au sens de Strauss et Corbin, 1990) et ne plus participer à la dérive des dirigeants et gestionnaires vers un éloignement du réel de l'activité.

Enfin, la distinction habituelle entre trois formes de prévention : «primaire», « secondaire» et « tertiaire » nous est apparue, chercheurs et acteurs de l'entreprise, pas aussi pertinente dans le domaine des «risques » psychosociaux que pour d'autres risques, chimiques, physiques, etc. ou pour une perspective hygiéniste. Nous souhaiterions, ainsi, revoir la signification des notions de préventions primaire, secondaire, tertiaire, sous l'angle d'une psychologie du travail et des organisations ainsi constituée :

- les interventions primaires correspondraient à des interventions sur l'organisation (global) et l'activité (local), avec des aller/retour, pour favoriser le développement du métier, aussi bien que celui de l'organisation, l'échelle organisationnelle permettant de repérer les groupes en difficulté et la clinique de l'activité d'agir auprès de ces groupes pour qu'ils retrouvent les voies de développement de leurs métiers ;

- la prévention secondaire pourrait s'adresser à l'encadrement, non pas pour lui apprendre à « gérer son stress », mais pour lui permettre de faciliter la création et le maintien de collectifs de travail, seuls à même de construire des solutions aux problèmes rencontrés dans le métier par le débat entre pairs. Caroly (2010) a montré, sur de nombreux exemples, l'importance de cette forme d'activité collective pour la santé des individus. Réinstaurer le débat entre pairs permettrait de construire plus solidement des marges de manœuvre dans l'action, que de simplement renforcer les capacités de faire face des individus ;

- la «prévention » tertiaire correspondrait à l'accompagnement, par des psychologues avertis, des enjeux du travail (connaissance de la clinique de l'activité et de la psychodynamique du travail), des individus sur le sens de leur travail, l'analyse de leurs contextes d'action, afin de retrouver le pouvoir d'agir, sans, nécessairement, faire un détour par l'enfance ou par l'acquisition de techniques d'adaptation.

\section{RÉFÉRENCES}

AUCOUTURIER (Valérie).- Les approches cliniques du travail dans la littérature anglo-saxonne, août 2010 [http://www.college-risquespsychosociaux-travail.fr/site/ Revue-psychologie-clinique-anglo-saxonne.pdf ; le 12/ 01/2011].

Bebessiki (Danièle).- Conséquences psychologiques et sociales du chômage partiel de longue durée, Mémoire de master, Institut de psychologie, Université Lyon 2, juin 2010 .

BourgeoIs (Fabrice), Gonon (Olivier).- Le lean et l'activité humaine, @ctivités, 7, 1, 2010, p. 136-142 [http://www.activites.org/v7n1/bourgeois.pdf].

CAROLY (Sandrine).- L'activité collective et la réélaboration des règles : des enjeux pour la santé au travail, Habilitation à diriger des recherches, Université Bordeaux 2, 3 mars 2010.
Clot (Yves).- Travail et pouvoir d'agir, Paris, Presses universitaires de France, 2008.

Clot (Yves).- Le travail à cour. Pour en finir avec les risques psychosociaux, Paris, La découverte, 2010.

Clot (Yves), Lhuilier (Dominique).-Agir en clinique du travail, Toulouse, Erès, 2010.

Cohen (Sheldon), Kamarck (Tom), Mermelstein (Robin).- A global measure of perceived stress, Journal of health and social behavior, 24, 1983, p. 385-396.

Coutrot (Thomas), Mermilliod (Catherine).- Les risques psychosociaux au travail : les indicateurs disponibles, DARES Analyses, 81, décembre 2010.

COX (Tom), TARIS (Toon), NiElsen (Karina).- Organizational interventions : issues and challenges, Work \& stress, 24, 3, 2010, p. 217-218. 
Digonnet (Laure).- Communiquer au travail. Étude de la communication entre managers et opérateurs dans un centre de fabrication, Mémoire de master, Institut de psychologie, Université Lyon 2, juin 2008.

Dugué (Bernard), Petit (Johann), Daniellou (François).- L'intervention ergonomique comme acte pédagogique, Pistes, 12, 3, 2010 [http://www.pistes.uqam.ca/ v12n3/pdf/v12n3a2.pdf]

DuPONT (Adeline).- Stress professionnel : juste une course contre le temps ?, Mémoire de maittrise, Institut de psychologie, Université Lyon 2, juin 2002.

Giga (Sabir), CoOper (Cary), FARAGHER (Brian).- The development of a framework for a comprehensive approach to stress management interventions at work, International journal of stress management, 10, 4, 2003, p. 280-296.

HANSEZ (Isabelle), BerTRAND (Françoise), BARbIER (Marie).-Évaluation des pratiques de diagnostic de stress au sein d'entreprises belges: facteurs bloquants et facteurs stimulants, Le travail humain, 72, 2, 2009, p. 127-153.

KARASEK (Robert), Theorell (Tores).- Healthy work : stress, productivity, and the reconstruction of working life, New York, Basic books, 1990.

Karnas (Guy), Hellemans (Catherine).- Approche psycho-organisationnelle du stress professionnel: les limites d'un modèle, dans Neboit (M.), Vézina (M.), Stress au travail et santé psychique, Toulouse, Octarès, 2002, p. 174-188.

KRIEF (Alexia).- L'utilisation de l'anglais au travail : étude des répercussions sur l'organisation du travail, Mémoire de master, Institut de psychologie, Université Lyon 2, juin 2010.

KRISTENSEN (Tage Sondergard).- A questionnaire is more than a questionnaire, Scandinavian journal of public health, 38, 2010, p. 149-155.

LAHLOU (Saadi).- Attracteurs cognitifs et travail de bureau, Intellectica, 30, 2000, p. 75-113.

Lamontagne (Anthony), Keegel (Tessa), Louie (Amber), Ostry (Aleck), LANDSBERGIS (Paul).- A systematic review of the job stress intervention evaluation literature, 1990-2005, International journal of occupational \& environmental health, 13, 3, 2007, p. 268-280.

LANCRY (Alain).- Incertitude et stress, Le travail humain, 70, 3, 2007, p. 289-305.

Lemyre (Louise), Tessier (Réjean), Fillion (Lise).Mesure du stress psychologique (MSP), Manuel d'utilisation, Québec, Behaviora, 1990.

LHUILIER (Dominique).- Travail, management et santé psychique, Connexions, 91, 1, 2009, p. 85-101.

Liu (Michel).- Fondements et pratiques de la recherche-action, Paris, L'harmattan, 1997.

MyKLEDUn (Arnstein), STORdAll (Eystein), DAHL (Alv).- Hospital Anxiety and Depression (HAD) scale : factor structure, item analyses and internal consistency in a large population, British journal of psychiatry, 179 , 2001, p. 540-544.

Niedhammer (Isabelle), Chastang (Jean-François), Gendrey (L), David (Simone), Degioanni (Stéphanie).Propriétés psychométriques de la version française des échelles de la demande psychologique, de la latitude décisionnelle et du soutien social du "Job content questionnaire" de Karasek: résultats de l'enquête nationale SUMER, Santé publique, 18, 3, 2006, p. 413-427.

Rouat (Sabrina).- Les processus individuels et organisationnels de construction de la santé au travail: prévention de la santé mentale au travail et analyse de dispositifs organisationnels, Thèse de doctorat en psychologie, sous la direction de A. Houel et Ph. Sarnin, Université Lumière Lyon 2, 14 janvier 2010.

Saint-Arnaud (Louise), Gignac (Sylviane), Gourdeau (Pierre), Pelletier (Mariève), Vézina (Michel).-Démarche d'intervention sur l'organisation du travail afin d'agir sur les problèmes de santé au travail, Pistes, 12, 3, 2010, [http://www.pistes.uqam.ca/v12n3/pdf/v12n3a4.pdf].

SARNIN (Philippe).- Mergers and acquisitions : dimensions of work related stress involved, $\mathrm{XI}^{\text {th }}$ European congress on work and organizational psychology, Lisbonne, 14-17 mai 2003.

SARnin (Philippe), Balas-Broche (Olivia).- Charges de travail et activités des cadres ingénieurs de bureaux d'études, dans Livian (Yves), Être cadre, quel travail?, Lyon, ANACT, 2006, p. 206-219.

Sarnin (Philippe), Fernandez (Sandra), Malaurie (Julia).- Rachat d'entreprise. Le vécu des salariés, dans Crescenzo (J.-C. de), Changement dans les organisations. Tome 2. Communication, négociation et intervention, Paris, L'harmattan, 2006, p. 169-181.

SIEGRIST (Johannes).- Adverse health effects of higheffort/low-reward conditions, Journal of occupational health psychology, 1, 1, 1996, p. 27-41.

SORNET (Gersende).- «J'e-mail donc je suis ». Étude des répercussions de la messagerie électronique sur l'activité des salariés, Mémoire de master, Institut de psychologie, Université Lyon 2, juin 2007.

STRAuss (Anselm).- La trame de la négociation, Paris, L'harmattan, 1992.

STRAuss (Anselm), Corbin (Juliet).- Basics of qualitative research: grounded theory procedures and techniques, Newbury Park, Sage, 1990.

VAleyre (Antoine).- Conditions de travail et santé au travail des salariés de l'Union européenne : des situations contrastées selon les formes d'organisation, Document de travail CEREQ, $n^{\circ} 73$, novembre 2006.

VÉZINA (Michel).- Stress et psychodynamique du travail : de nouvelles convergences Travailler, 2, 1999, p. 201-218. 